Voix et Images

voixetimages

\title{
Le théâtre dans tous ses états
}

Lucie Robert

Volume 24, numéro 1 (70), automne 1998

Yves Préfontaine

URI : https://id.erudit.org/iderudit/201418ar

DOI : https://doi.org/10.7202/201418ar

Aller au sommaire du numéro

\section{Éditeur(s)}

Université du Québec à Montréal

\section{ISSN}

0318-9201 (imprimé)

1705-933X (numérique)

Découvrir la revue

\section{Citer cet article}

Robert, L. (1998). Le théâtre dans tous ses états. Voix et Images, 24(1), 207-214. https://doi.org/10.7202/201418ar d'utilisation que vous pouvez consulter en ligne.

https://apropos.erudit.org/fr/usagers/politique-dutilisation/ 


\title{
Dramaturgie
}

\section{Le théâtre dans tous ses états}

\author{
Lucie Robert, Université du Québec à Montréal
}

S'il est une qualité à la prolifération des manuels scolaires portant sur la littérature québécoise, à peu près tous rédigés pour l'enseignement collégial, c'est bien celle d'avoir renouvelé l'intérêt pour les grands textes qui la composent. À côté des synthèses historiques et des anthologies, où l'on continue à parler des textes dramatiques avec beaucoup de précautions ${ }^{1}$, certaines maisons d'édition ont ainsi créé ces derniers temps des collections spécialisées d'explication de texte préparées dans la tradition la plus conventionnelle du genre. Les programmes d'enseignement ont leur logique propre qui distinguent cette activité d'une réflexion plus liée à la recherche et ils imposent tant une manière de faire qu'un objet. Aussi a-t-on vu paraître récemment, dans ces collections scolaires, trois études portant sur des textes dramatiques. C'est peu, mais force est de constater qu'il s'agit des seuls ouvrages à avoir été consacrés entièrement à Un simple soldat de Marcel Dubé ${ }^{2}$, aux Belles-sours ${ }^{3}$ et à $\dot{A}$ toi, pour toujours, ta Marie-Lou de Michel Tremblay ${ }^{4}$.

Évidemment de telles études n'ont que peu à voir avec de véritables essais critiques qui supposent un choix de perspective et de méthode, une démonstration centrée sur une problématique unique sinon englobante et une véritable pensée. Quel que soit son rapport à l'activité scien- tifique, l'essai critique recherche l'originalité et il laisse au sujet écrivant le soin d'explorer une problématique en profondeur. Dans ce cas-ci, nous sommes loin d'une telle attitude et les auteurs n'ont pour s'exprimer que la latitude que leur laissent les contraintes de la didactique et celles de la collection dans laquelle ils publient. La lecture est décomposée en fragments: "auteur", "contexte", "structure et composition", "thèmes et motifs", "fond et forme", et est accompagnée de résumés "à retenir", d'une brève "bibliographie", d' "extraits critiques" et de "sujets et plans de dissertation". Une telle organisation n'est pas sans entraîner certaines répétitions, mais elle a le mérite de sortir les élèves des habituelles analyses de contenu qui débouchent inévitablement sur la comparaison entre la fiction et le monde réel. Chaque collection construit son modèle propre, mais le programme d'enseignement les contraint toutes à envisager l'œuvre sous des angles prédéterminés.

De l'étude que Michel Laurin propose du texte de Marcel Dubé, on ne dira pas grand-chose, sinon qu'elle montre tous les défauts du genre, se repliant sur une conception conservatrice de l'analyse littéraire et n'accordant que peu d'importance aux particularités du texte dramatique traité ici comme un roman, c'est-à-dire beaucoup réduit à son 
anecdote. La lecture proposée du texte de Dubé est essentiellement centrée sur l'analyse des personnages, avec un fort penchant pour le psychologisme, et sur la comparaison entre l'histoire propre du personnage de Joseph Latour et celle de la collectivité québécoise, poncif désespérément ressassé depuis trop longtemps. Tout n'est cependant pas mauvais dans cette étude, on notera que la notice biographique est bien informée; que le contexte socio-historique, sans être dénué de parti pris, reste efficacement brossé; que les quelques pages sur la langue de Marcel Dubé sont bien conçues et que la comparaison avec les autres œuvres de l'époque propose d'étonnants et fructueux croisements:

Plus intéressante est la conception de l'analyse littéraire proposée par la collection de Boréal, "Les classiques québécois expliqués ", qui montre un. plus grand souci de la forme que celle de la collection Beauchemin que dirige Michel Laurin et qui conserve un plan plus traditionnel où dominent encore les thèmes, les personnages et l'espace. Les deux études consacrées à Michel Tremblay publiées dans cette collection, celle d'Yves Jubinville sur Les belles-sœurs et celle de Louise Vigeant sur $\bar{A}$ toi, pour toujours, ta Marie-Lou, ont toutes deux été publiées par des spécialistes de thêâtre et elles sont remarquablement bien informées des contraintes particulières de l'analyse des textes dramatiques.

La lecture que propose Yves Jubinville des Belles-sœurs de Michel Tremblay est fine et attentive. Visiblement au courant des réflexions les plus récentes sur l'écriture dramatur- gique, l'auteur cerne avec une grande efficacité l'originalité du texte de Tremblay qu'il situe au croisement de trois traditions: la tradition populaire propre à Gratien Gélinas à laquelle il emprunte le caractère festif et comique, le théâtre de répertoire d'où il tire les sujets sérieux et les références savantes (comme la tragédie) et le théâtre d'avant-garde ou d'expérimentation qui renouvelle les procédés dramaturgiques et scéniques et qui adopte, devant le monde, un ton ironique. Sans entrer dans le détail de l'analyse, ce que ce type d'ouvrage ne permet pas, l'auteur dissèque l'écriture de Tremblay en montrant ce qui, dans le joual, est une langue construite; il distingue entre le dialogue et le bavardage, analyse les divers usages du monologue et précise la fonction variable des chours dans l'économie de la pièce. Jubinville revient sur les grandes métaphores structurantes de la pièce (la messe, la communion et le Théâtre du monde). De l'analyse des thèmes et motifs, où sont mis en opposition la religion et la consommation, la culture savante et la culture populaire, l'individu et le groupe, il confirme une interprétation d'ensemble introduite dès le départ: "Les belles-sœurs montre l'envers du décor ou encore les coulisses de la Révolution Tranquille." (p. 27) Bref, on sort de cette lecture avec l'impression d'avoir appris quelque chose de nouveau, non seulement à propos des Belles-sœurs, mais à propos du théâtre contemporain, et doté de nouveaux moyens pour lire le théâtre. L'enseignement de la littérature au cégep a de l'avenir. Il y a là assez d'idées neuves pour nous faire regretter l'essai critique que Jubinville aurait pu écrire 
et qu'il n'écrira sans doute plus sur cette pièce.

Évidemment, pour qui a lu ces ouvrages dans l'ordre de leur parution - ce que j'ai malheureusement fait -, il devient difficile d'apprécier à sa juste valeur l'ouvrage de Louise Vigeant. D'autant que Jubinville, plus jeune, m'était moins connu. Je lui savais des chroniques, parues dans Spirale et dans Jeu, et de rares articles, mais aucun ouvrage d'envergure à part celui-ci, si tant est que l'on puisse parler d'envergure à propos de ce petit livre. Au contraire, la pensée de Louise Vigeant a trouvé depuis de nombreuses années à se déployer de diverses manières. On rappellera notamment son ouvrage La lecture $d u$ spectacle théâtral ${ }^{5}$, écrit pour les étudiants de niveau collégial à une époque où personne ne songeait à le faire, et qui offre, en guise d'exemple, une solide lecture de Vie et mort $d u$ roi boiteux de Jean-Pierre Ronfard. Davantage animée par une sémiologie théâtrale que par une sémiotique du texte dramatique, Vigeant est moins à l'aise que Jubinville dans la lecture d'À toi, pour toujours, ta Marie-Lou. C'est ainsi dans l'analyse plus conventionnelle du dialogue, de l'espace dramatique et du système des personnages qu'elle excelle, bien que l'utilisation répétée du modèle d'analyse mis au point par Greimas ait désormais un côté vieux jeu, du moins pour des lecteurs informés, car il conserve un certain pouvoir descriptif parfois utile. La structure musicale de l'œuvre est clairement exposée et son caractère tragique est justement précisé. L'entrelacement des dialogues et la structuration des répliques (monologues, tirades, silences) fait l'objet d'une analyse serrée qui montre admirablement la solitude des personnages. En revanche, l'étude des thèmes et motifs est plus conventionnelle (il faut dire que, sur ces questions, la pièce de Tremblay a été souvent commentée), centrée sur l'opposition entre le bonheur et le malheur, la liberté et l'aliénation. Néanmoins, l'auteure fait le point sur cette pièce et sa lecture en convaincra plusieurs. J'aurais pour ma part souhaité une lecture plus audacieuse, mais Louise Vigeant connaît ses lecteurs mieux que moi.

\section{$* *$}

L'audace n'est précisément pas ce qui fait défaut à Pascal Riendeau qui, avec La cobérence fautive ${ }^{6}$, publie le premier ouvrage sur Normand Chaurette. Le titre est emprunté à une réplique de Provincetown Playbouse, et il renvoie au caractère postmoderniste de l'œuvre de Chaurette dont l'écriture multiplie les effets non pas d'incohérence mais bien de "cohérence fautive", quand il brouille les genres, les distinctions entre didascalies et dialogues, quand il propose deux versions de la même scène, trois âges du même personnage, quand il insère des passages narratifs dans un texte dramatique ou, inversement, un texte dramatique au cœur du roman. J'avais éprouvé un grand plaisir de lecture en prenant connaissance de la première version de cette étude alors qu'elle était déposée comme mémoire de maîtrise à l'Université du Québec à Montréal, et j'ai retrouvé le même bonheur en lisant cette nouvelle version, un peu modifiée et mise à jour, que publie Nuit blanche éditeur. On y trouve à la fois une 
solide érudition dans la présentation des diverses approches du postmodernisme littéraire et de la sémiotique du texte dramatique ici mises à l'épreuve, une grande rigueur de l'analyse qui porte sur l'hybridité en tant que stratégie textuelle privilégiée par Chaurette dans sa pièce Provincetown Playbouse, juillet 1919, j'avais 19 ans et dans son roman Scène d'enfants, en même temps qu'une recherche d'écriture qui nous épargne tout effet scolaire et qui prend visiblement plaisir à balayer les lieux communs de la critique. On en redemanderait, en particulier sur les autres œuvres de Chaurette, trop rapidement introduites et situées en regard de la problématique générale, et sur les procédés dramaturgiques, évidemment un peu mis de côté, qui forment pourtant le style particulier de cet auteur en train de devenir un des écrivains majeurs de la littérature québécoise contemporaine.

\section{***}

Je suis toujours frappée par un fait simple et, somme toute, assez prévisible: s'agissant d'étudier les textes dramatiques, les spécialistes de théâtre et les autres spécialistes de littérature instituent des canons étrangers l'un à l'autre. Pour un Michel Tremblay, dont le statut dans la littérature québécoise fait consensus, combien d'autres restent méconnus. Combien de spécialistes du roman ou de la poésie songeraient spontanément à situer Normand Chaurette parmi les grands écrivains québécois? Tel est le premier étonnement éprouvé à la lecture de Nouveaux regards sur le théâtre québécois, actes du sixième colloque de l'Association des professeurs des littératures acadienne et québécoise de l'Atlantique, publiés par Betty Bednarski et Irene Oore $^{7}$. On n'y trouve précisément rien sur Chaurette. En fait, comme c'est généralement le cas dans ce genre d'ouvrage, chacun poursuit l'objet de ses réflexions antérieures à propos du thème général qui réunit les diverses contributions. Cela permet parfois d'heureuses rencontres.

Le théâtre d'Anne Hébert, de Marie-Claire Blais ou de Jacques Ferron, sauf pour Les grands soleils, n'est pas assez important dans l'histoire du théâtre québécois pour faire l'objet d'études spécialisées. Il l'est pourtant ici, sous la signature respective de Neil B. Bishop, Irene Oore et Pierre Gobin, bien que ces études enrichissent davantage notre connaissance de ces auteurs que de l'écriture dramaturgique proprement dite. Inversement et faute de textes, Pol Pelletier et Robert Lepage sont absents des colloques littéraires. Ils sont néanmoins présents dans le cadre d'une étude plus générale que Josette Féral réalise sur "La place des femmes dans les théories actuelles du jeu théâtral " et dans la lecture que Marta Dvorak effectue des Sept branches de la rivière Ota et d'Elseneur. La volonté des organisatrices d'ouvrir le colloque à la littérature de jeunesse incite Claire Lebrun à entreprendre ce que je crois être la première lecture d'ensemble de l'œuvre dramaturgique de Jasmine Dubé. Trois autres communications sont issues de thèses de doctorat en cours ou récemment soutenues. Elles portent toutes trois sur des questions de réception, diversement envisagées. Janusz Przychodzeń étudie l'horizon d'attente dans le champ théâtral à 
partir de l'attitude qu'ont les théâtres envers le spectateur; Daniel Chartier propose une lecture de la critique qui entoure les pièces d'Yvette Ollivier Mercier-Gouin tandis que Louise Ladouceur examine le théâtre anglo-canadien traduit au Québec depuis 1990. Parmi les textes canoniques de la dramaturgie actuelle, trois sont étudiés de manière attentive: Anthony M. Watanabe analyse les effets de la mise en abyme dans $L e$ vrai monde? de Michel Tremblay, Robert Viau s'intéresse à "La quête de la vérité dans Oublier de Marie Laberge", alors que Piet Defraye et Marylea MacDonald réévaluent la structure par enchâssement des Feluettes de Michel Marc Bouchard.

Je soulignerai l'intérêt particulier de deux communications. Pierre L'Hérault poursuit ses travaux sur la représentation de la diversité culturelle dans le théâtre québécois depuis 1977. "L'espace immigrant et l'espace amérindien dans le thêatre québécois depuis 1977" prend ainsi la suite d'un article portant sur les figures de l'immigrant et de l'Amérindien dans le même corpus, paru en 1996 dans la Revue internationale d'études canadiennes. De même, le "Daniel Danis et la dramaturgie de la parole" de Jane Moss, qui porte sur Celle-là, comprend un second volet portant sur Cendres de cailloux, publié dans Dalhousie French Studies au printemps de 1998, et fait suite à un article sur le même sujet, mais qui se penche sur l'œuvre de Larry Tremblay paru dans L'annuaire théâtral, au printemps de 1997. Ces deux recherches d'envergure méritent que l'on n'en perde pas le fil.

Enfin, je m'en voudrais de ne pas attirer l'attention sur les deux conférences d'André Ricard qui ouvre et ferme le livre. La première, "Pratique actuelle de la scène au Québec", permet à l'auteur de tracer sa propre histoire du théâtre québécois depuis 1970 et de montrer comment, du point de vue d'un dramaturge, se vit le conflit avec le metteur en scène. Pour Ricard, qui tient à réaffirmer "l'appartenance du théâtre à la littérature" (p. 201), la création en 1996, à quelques mois d'intervalles, du Passage de l'Indiana de Normand Chaurette au Festival d'Avignon dans une mise en scène de Denis Marleau, et du Voyage $d u$ couronnement de Michel Marc Bouchard, au Théâtre du Nouveau Monde, signale peut-être "une reprise de foi en la découverte de la part des grandes structures de production " (p. 21) et, à ce titre, elles ouvriraient une nouvelle période de l'histoire du théâtre québécois.

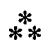

Tout autre est l'avis de Madeleine Greffard et de Jean-Guy Sabourin qui, au contraire de Ricard, célèbrent les années récentes de l'histoire du théâtre parce qu'elles ont "permis aux gens de thêâtre de s'imposer comme des gens de métier qui entendent faire du théâtre un art et une industrie" (p. 108). Leur ouvrage Le théâtre québécois ${ }^{8}$ est tout entier orienté vers la démonstration de cette idée et la valorisation de ce qui, depuis 1606 , tend à instituer l'activité théâtrale comme pratique autonome. Rédigé selon le modèle propre à la collection "Boréal express", qui se veut une version québécoise des "Que sais-je?" français, l'étude n'en est pas moins une synthèse générale et ma foi! 
assez complète de l'histoire du théâtre québécois. C'est une réalisation remarquable, s'il en est, que de tout signaler en cent vingt pages et de donner à lire, en particulier sur les quarante dernières années, un bon nombre de renseignements inédits et d'analyses nouvelles, en particulier sur les structures organisationnelles dont se sont dotés les praticiens. Une attention particulière est portée à tout ce qui permet de mieux saisir le mouvement de professionnalisation qui anime la scène depuis la fin du $\mathrm{XIX}^{\mathrm{e}}$ siècle, et en particulier aux divers regroupements professionnels qui assurent la pérennité des métiers de la scène depuis une trentaine d'années. Là est d'ailleurs le fil conducteur principal de l'ouvrage puisque les auteurs postulent que la professionnalisation de ces métiers est l'indice principal d'une activité durable et autonome.

En conséquence, l'étude porte presque exclusivement sur l'activité professionnelle, le théâtre amateur n'ayant à ce titre que peu d'importance. Toutefois, et parce l'histoire du théâtre professionnel n'offre pas de perspective continue (elle connaît trop d'interruptions), Greffard et Sabourin accordent une certaine attention à des manifestations amateurs qui sont à l'origine des pratiques professionnelles. C'est le cas par exemple des Compagnons de Saint-Laurent entre 1937 et 1952 ou du jeune théâtre autour de 1970. Pour distinguer ces manifestations du théâtre amateur proprement dit (le théâtre de collège ou les cercles dramatiques), les auteurs construisent une catégorie particulière d'événements qu'ils nomment malheureusement les "non-professionnels". La désignation d'une catégorie de ce genre par la négative est toujours un peu gênante et, à mon avis, elle dénote plus un manque d'analyse qu'autre chose. D'autant que les auteurs rendent la notion opérationnelle pour les années modernes (depuis 1937), mais qu'ils négligent entièrement de considérer le travail réalisé sous le même angle au $x_{\text {viII }}{ }^{e}$ et XIX $^{e}$ siècle par Joseph Quesnel, Napoléon Aubin et Louis Fréchette. Toutefois, il serait injuste de leur en vouloir de n'avoir pas su, mieux que les autres chercheurs, résoudre cette équation motrice de l'histoire du théâtre québécois. Il faudrait plutôt saluer chapeau bas cet ouvrage, premier essai de synthèse couvrant l'histoire du théâtre au Québec depuis la Nouvelle-France jusqu'à nos jours à paraître depuis quarante ans, c'est-àdire depuis les Trois cent cinquante ans de théâtre au Canada français de Jean Béraud.

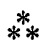

"En 1912, Julien Daoust, qui a dirigé entre-temps le Théâtre Populaire, place Jacques-Cartier à Québec, s'installe à Montréal dans une ancienne salle de cinéma, le Nationoscope, qu'il rebaptise le Canadien. (p. 24), écrivent Greffard et Sabourin. C'est tout ce que nous saurons de ce qui, du point de vue de la ville de Québec, a constitué une sorte d'"âge d'or" et que Christian Beaucage décrit comme "Une période flamboyante", selon le sous-titre qu'il donne à son ouvrage portant sur Le théâtre à Québec au début du $x x^{e}$ siècle? Renouant avec les grands travaux de recherche fondamentale qui, comme ceux de Jean-Marc Larrue sur Montréal, visent à reconstituer l'acti- 
vité théâtrale d'une ville, d'une troupe ou d'une région - travaux qui depuis vingt ans ont considérablement renouvelé notre lecture de l'histoire - Beaucage retrace, théâtre par théâtre et année par année, les événements théâtraux de la ville de Québec. Lui aussi se concentre sur les activités professionnelles, mettant de côté le théâtre de collège. Ses renseignements lui viennent du dépouillement des journaux francophones de l'époque, Le Soleil et L'Événement.

L'étude est circonscrite par deux événements majeurs: l'incendie de l'Académie de musique, de la hauteville, en 1900 , et celui du Théâtre populaire, de la basse-ville, en 1911. Théâtre de prestige, l'Académie de musique avait été le témoin de tous les grands événements théâtraux de la seconde moitié du $\mathrm{XIX}^{\mathrm{e}}$ siècle. Sa disparition, montre Beaucage, entraîne le déplacement du théâtre francophone vers la basse-ville. $\mathrm{Ne}$ resteront à la haute-ville, au Tara Hall puis à l'Auditorium, que les tournées en provenance des États-Unis et les spectacles de musique ou de variétés. C'est donc à la salle JacquesCartier, rue Saint-Joseph, que les choses se passent: Léon Petitjean, Georges Gauvreau, Lucien Prad, Wilfrid Villeraie, Palmiéri et Blanche de la Sablonnière sont parmi les directeurs qui s'y installent le temps d'une ou plusieurs saisons pour y mettre à l'affiche un répertoire essentiellement consacré au mélodrame. Julien Daoust y triomphe, avec Bella Ouellette, et c'est à Québec qu'il se refait une réputation de directeur de théâtre, la sienne ayant été entachée à Montréal par les malheurs du Théâtre National. Il y poursuit le même projet, celui de mettre sur pied un théâtre comparable à la ComédieFrançaise, et il y crée un répertoire reposant sur les mêmes choix, privilégiant le mélodrame (la comédie ne connaît guère de succès à l'époque chez les francophones), mais accordant néanmoins une attention toute spéciale aux œuvres du cru. Son travail, pour le développement du théâtre dans la ville de Québec, est aussi capital qu'il ne l'est dans l'histoire de Montréal.

Les calendriers et listes de représentations reconstitués par Beaucage occupent près de la moitié du livre. L'analyse qui les précède est divisée en quatre parties portant respectivement sur les salles, les acteurs et troupes, les genres et formes ainsi que les fonctions sociales de ce théâtre. Nous sommes dans un récit qui emprunte sa dynamique à une sociologie descriptive du théâtre. Bien que l'information domine l'analyse et que tous les arbres retrouvés nous fassent parfois perdre de vue la forêt, l'étude de Beaucage se lit aisément comme l'épopée interrompue du théâtre à Québec. En 1911, en effet, après l'incendie de la salle Jacques-Cartier, c'est à Montréal que se réinstalle Julien Daoust. À demeure.

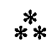

Au cours des trois premiers siècles de l'histoire du théâtre québécois, aucun critique n'aura soulevé autant de passions que Robert Lévesque. Honni et dénoncé plusieurs fois par le milieu, il persiste et signe pour mon plus grand plaisir $L a$ liberté de blâmer. Carnets et dialogues sur le théâtre ${ }^{10}$, préparé avec la collaboration de Stéphane Lépine, à partir des entrevues et textes lus et 
diffusés sur les ondes de la chaîne MF de Radio-Canada à l'automne de 1996. Lévesque venait alors tout juste de quitter Le Devoir où il avait signé un nombre considérable de critiques depuis 1980 (avant, on avait pu le lire dans Québec-Presse et dans Le Jour). On se rappellera, comme le haut fait de cette période, la pétition de 1984, signée par 156 professionnels du théâtre, exigeant de la direction du journal qu'elle renvoie son critique. La pétition eut l'effet inverse, c'est-à-dire que Lévesque acquit alors son statut de critique permanent. Il y aura une autre pétition, en 1992, à peu près au moment où il devient directeur des pages artistiques du journal.

Robert Lévesque est un être trouble qui ne mâche pas ses mots et ne prend aucun détour pour faire comprendre ce qu'il a à dire. Cela le rend insupportable et je dois dire que le dernier carnet, intitulé "Je me souviens", contient des souvenirs qui ne lui attireront aucun capital de sympathie. Pas même le mien. Toutefois, l'ensemble des réflexions que contiennent les autres carnets montre une connaissance approfondie de l'activité thêatrale contemporaine et une passion pour celle-ci que rien n'a démentie. Lévesque fustige d'abord la paresse, l'ignorance et la complaisance. Dieu sait qu'il y en a dans le milieu artistique! De plus, il n'a guère apprécié la grande période historicopolitique du théâtre des années soixante-dix et il garde rigueur à Jean-Claude Germain qui le lui rend bien. En revanche, il rappelle certains moments de pure magie : la représentation du cycle intégral de Vie et mort du roi boiteux de Jean-Pierre Ronfard à la Cité du Havre de Montréal en juin 1982; la représentation du Soulier de satin, de Paul Claudel à Avignon en juillet 1987, dans une mise en scène d'Antoine Vitez. Et il trace son palmarès: Lepage, Maheu, Marleau, Chaurette, Tremblay, "Dyne, Pol, Patricia et cette chère Sylvie", "Béland, Lebeau, Millette et les autres". Il ne se fera pas que des amis, mais peut-on lui donner tort? Quand le langage critique exprime autant de passion, on doit pouvoir lui pardonner sa hargne.

1. "La dramaturgie est-elle (encore) un genre littéraire?" demandent d'entrée de jeu Bernard Andrès et Pascal Riendeau dans Panorama de la littérature québécoise contemporaine, sous la direction de Réginald Hamel, Montréal, Guérin, 1997, p. 208.

2. Michel Laurin, Étude de Un simple soldat de Marcel Dubé, Montréal, Groupe Beauchemin éditeur, coll. "Parcours d'une neuvre", 1998, $87 \mathrm{p}$.

3. Yves Jubinville, Une êtude de Les bellessœurs de Micbel Tremblay, Montréal, Boréal, coll. "Les classiques québécois expliqués ", 1998, 115 p.

4. Louise Vigeant, Une étude de À toi, pour toujours, ta Marie-Lou de Michel Tremblay, Montréal, Boréal, coll. "Les classiques québécois expliqués ", 1998, 129 p.

5. Louise Vigeant, La lecture du spectacle théâtral, Laval, Mondia, 1989.

6. Pascal Riendeau, La cobérence fautive. L'bybridité textuelle dans l'ceuvre de Normand Chaurette, Québec, Nuit blanche éditeur, coll. "Études", 1997, 164 p

7. Betty Bednarski et Irene Oore [dir.], Nouveaux regards sur le théâtre québécois, Montréal/Halifax, XYZ éditeur/Dalhousie French Studies, coll. "Documents", 1997, 203 p.

8. Madeleine Greffard et Jean-Guy Sabourin, Le théâtre québécois, Montréal, Borẹal, coll. "Boréal express ", 1997, 121 p.

9. Christian Beaucage, Le théâtre à Québec au début $d u x^{e}$ siècle. Une époque flamboyante!, Québec, Nuit blanche éditeur, coll. "Les Cahiers du CRELIQ, Série Études ", 1996, $318 \mathrm{p}$.

10. Robert Lévesque, La liberté de blâmer. Carnets et dialogues sur le théâtre, Montréal, Boréal, coll. "Papiers collés", 1997, 194 p. 